\title{
A Prototype Rule-Based Expert System for Travel Demand Management
}

\author{
Resdiansyah Mansyur $^{1}$, Riza Atiq O.K Rahmat ${ }^{2}$ and Amiruddin Ismail ${ }^{3}$
}

\begin{abstract}
In this research, a prototype rule-based expert system for travel demand management (TDM) in selecting suitable policy was designed and developed. This expert system suggests a process for travel demand management policy implementation, and offers guidance and advice on the selection of effective and appropriate policies. The system is supported with an objectoriented modeling in the knowledge abstraction and representation, and the interactions with the system are performed through a friendly graphical user interface (GUI). The prototype of the system was developed in shell expert system platform (KAPPA-PC). The outcomes obtained by the current prototype show that the used rule-based expert system architecture resulted in successful computerized intelligent decision support software that is easy to maintain, modify, and extend.
\end{abstract}

Keywords: expert system, artificial intelligent, travel demand, object-oriented modeling

\section{INTRODUCTION}

$\mathrm{D}^{\mathrm{B}}$ EMAND on travelling increased with the assumptions that private car is the best or the only solution for mobility. The supply for the infrastructure increase proportional to traffic volume. At one point, supply cannot meet demands due to certain factors such as budget, land or space issue [1]. It is increasingly become accepted that unrestrained travel by private car within cites cannot be accommodated. This is due to a combination of financial constraints and concerns about the adverse impact of traffic on local communities and their environment. Allowing traffic to grow to levels at which there is extensive and regular congestion is economically inefficient [2]. Although the construction of additional road capacity can alleviate some of the effects of congestion, the benefits may be counterbalanced unless growth in traffic volumes can be restrained. Therefore, another alternative is by implementing Travel Demand Management (TDM) policy in order to make sure that people still travel but at the same time reducing the private car usage [1]. The implementation of TDM requires an expert, expertise or adviser person because with their knowledge, they can give advice on the most suitable TDM policies based on the purpose and location details. Unfortunately, there are very few numbers of expert persons that can be referred to give advice, and they are most likely not available all the time. Experts are not always available, nor do they always have the time to consult all possible references, review available data, etc [3]. This will lead towards time consuming on scheduling appointments and interviews, thus delaying one project. Later, it is a burden for Junior Engineers to refer books as their advisers to seek solution on implementing TDM [3]. As a result, many TDM programs are ineffective or were developed by an inefficient process of trial and error. Therefore, development of a rule-based expert system (ES) in TDM to assist junior transport engineer and the policy maker in selecting effective and appropriate TDM would be a valuable tool.

\section{EXPERT SYSTEM}

A computer tool used to develop the expert system is rule-based expert system software (Kappa-PC version 2.4). It is initial version of shell ES described by Intellicorp [4]. Selection of Kappa PC software is due to suitability in designer mode, which gives the knowledge engineer the possibility to code the knowledge base in a graphical (easily readable and editable) way that follows the latest trends in user interface. It lets the engineer decide what method to use for coding the knowledge [5], thereby addressing more complex problems [6]. An expert system shell is a software development environment containing the basic components of expert systems. Associated with a shell is a prescribed method for building applications by configuring and instantiating these components [7]. Some of the generic components of a shell are shown in figure 1 and described below.

\footnotetext{
${ }^{1}$ Resdiansyah Mansyur is with the Universiti Malaysia Sarawak, Kota Samarahan, 94300 Sarawak Malaysia (phone: 60-82583222; fax: 60-82583410; e-mail: mresdiansyah@feng.unimas.my).

${ }^{2}$ Riza Atiq O.K Rahmat is with Sustainable Urban Transport Research Center (SUTRA), Universiti Kebangsaan Malaysia, 43600, Selangor, Malaysia (e-mail: riza@vlsi.eng.ukm.my).

${ }^{3}$ Amiruddin Ismail is with Sustainable Urban Transport Research Center (SUTRA), Universiti Kebangsaan Malaysia, 43600, Selangor, Malaysia (email: abim@vlsi.eng.ukm.my).
} 


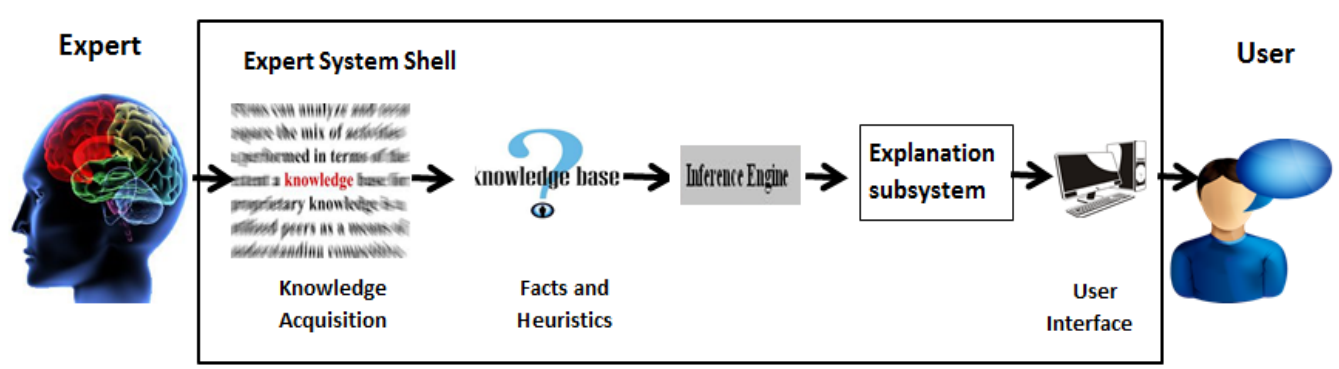

Figure 1 Generic component of shell system

Expert system (ES) can be defined as a program that attempts to mimic human expertise by applying inference methods to a specific body of knowledge [8]. Given the number of textbooks, journal articles, and conference publications about expert systems and their application, it is not surprising that there exist a number of different definitions for an expert system. Pomykalski [9] stated that an expert system is a computer program that is designed to mimic the decision-making ability of a decision-maker(s). Many agree that the expert system area has advanced furthest and achieved the most success in applying artificial intelligent methods to real world problem [10]. Expert system is a very successful application of artificial intelligence (AI) technology [11]. The common term for a system that uses AI is intelligent system or automated system.

Therefore, the research requires developing an expert system as a replacement for expert person in selection of TDM policies. The system developed has successfully provided most appropriate policies and the potential impacts in order to support decision making by young transport planner. The impacts involve benefit, equity and travel impact. Since it is an expert advisory system, the final advice has been given in a form of options which allows the user i.e. junior transport planner, to choose based on suitability in implementing of TDM

\section{SYSTEM ARCHITECTURE AND RULE-BASED REPRESENTATIVE PROCEDURE}

Various methods has been conducted which were data collections from the experts domain such as books, journals and human expert (knowledge acquisition), selection policies of TDM and rule-based representative procedure (IF-THEN) as programming tools inside of inference engine of expert system.

A computer tool used to develop the expert system is rule-based expert system software (Kappa-PC 2.4 version 2.4). Kappa-PC is a system which combines tools and methods from knowledge engineering with a high level graphical environment [4]. Knowledge was represented in Kappa-PC using frame-like objects that are organized in a hierarchy. KappaPC provides the developer with the flexibility and power required in supporting complex applications. It allows the knowledge engineer to develop prototype using rule-based knowledge representation technique. Running under windows environment, Kappa-PC provides a resources editor for developing interactive graphical user interfaces to applications. The graphical images created for each application are stored in the object hierarchy and so are within the object oriented frame work of the system. The hierarchy structure for TDM policies towards specific objectives, main purpose, geographical area and the final selected three advices shows as example in figure 2 below.

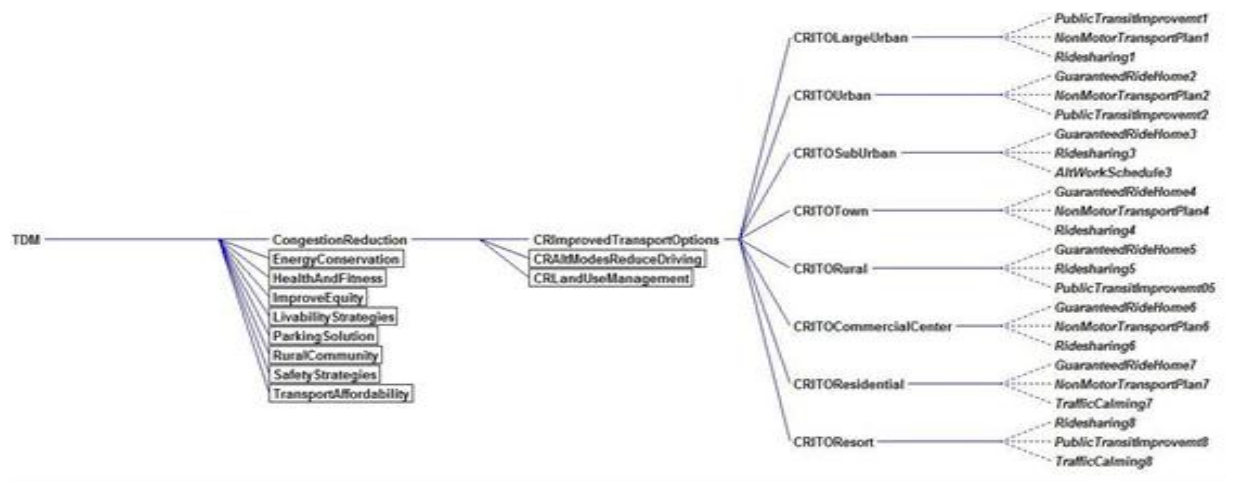

Figure 2 Example of Hierarchy Architecture for domain area 


\section{DEVELOPMENT OF EXPERT SYSTEM}

As an illustration of typical object representation in the rule-based expert system of TDM policy, the hierarchy of CRimprovedtransportoption class which is a descendant of the CongestionReduction class is shown in Figure 3.

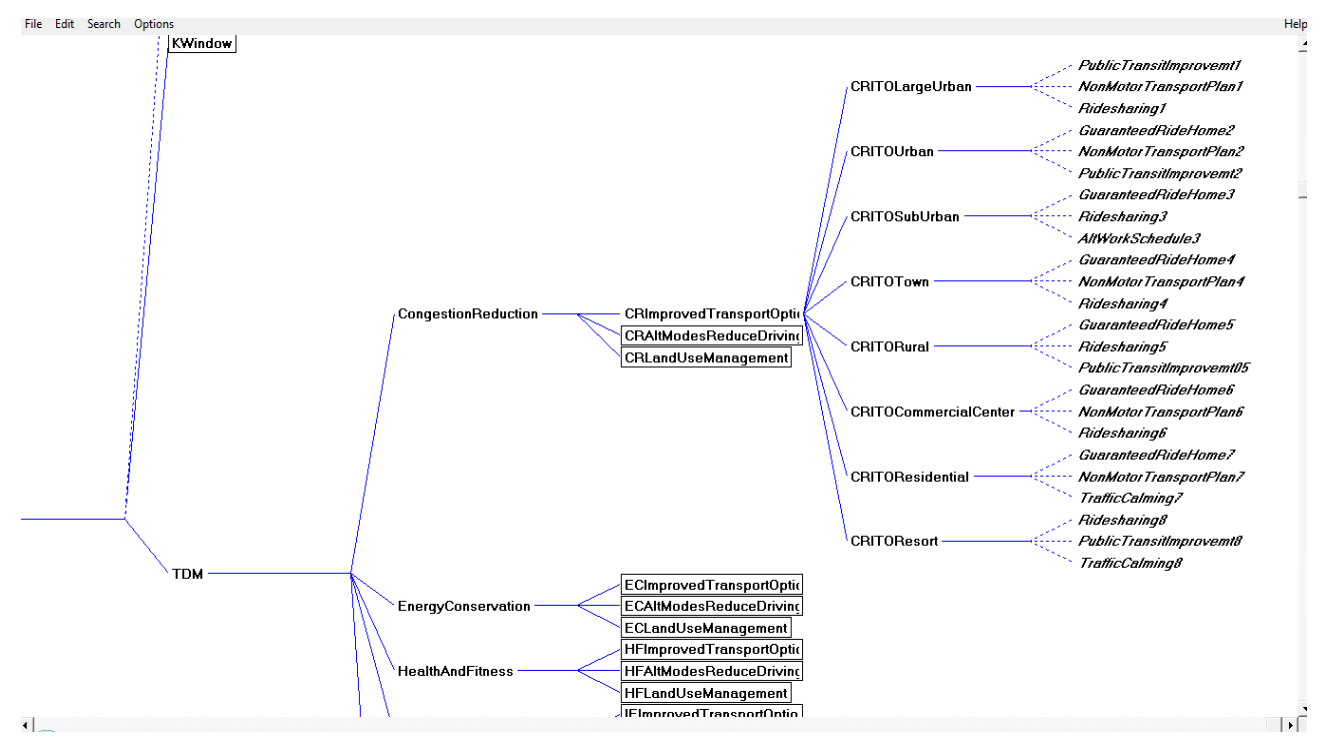

Figure 3 Illustration of hierarch method

CRITOLargeUrban class is geographical area entity that represents appropriate condition of area need to be implementing TDM strategies. It contains the slots: (i) PublicTransportImprovement1, which indicates the strategy on Public Transit (also called Mass-Transit) includes various services using shared vehicles to provide mobility to the public; (ii) Non Motor Transport Plan1, which indicates Non-motorized Transportation (also known as Active Transportation and Human Powered Transportation) includes walking and bicycling, and variants such as Small-Wheeled Transport (skates, skateboards, push scooters and hand carts) and Wheelchair travel; (iii) Ridesharing1, which refers to carpooling and vanpooling (the term is sometimes also applied to public transit, particularly commuter express bus), in which vehicles carry additional passengers. Carpooling uses participants' own automobiles. Vanpooling usually uses rented vans (often supplied by employers, nonprofit organizations or government agencies). Most vanpools are self-supporting - operating costs are divided among members. Vanpooling is particularly suitable for longer commutes. These slots are inherited from the parent class CRimprovedtransportoption which is a descendant of the CongestionReduction class.

\section{THE USER INTERFACE AND SAMPLE CONSUlTATION}

The E-ASSIST knowledge base is made up of nine class module, each deal with specific strategy of TDM domain. First, launch the system by opening the Kappa-PC software and open the file (.kal) to start the expert system. After the program is loaded, the general information interface appears (figure 4) in the center of the displaying area if the computer has resolution of $1366 \times 768$ pixel (32 bits).

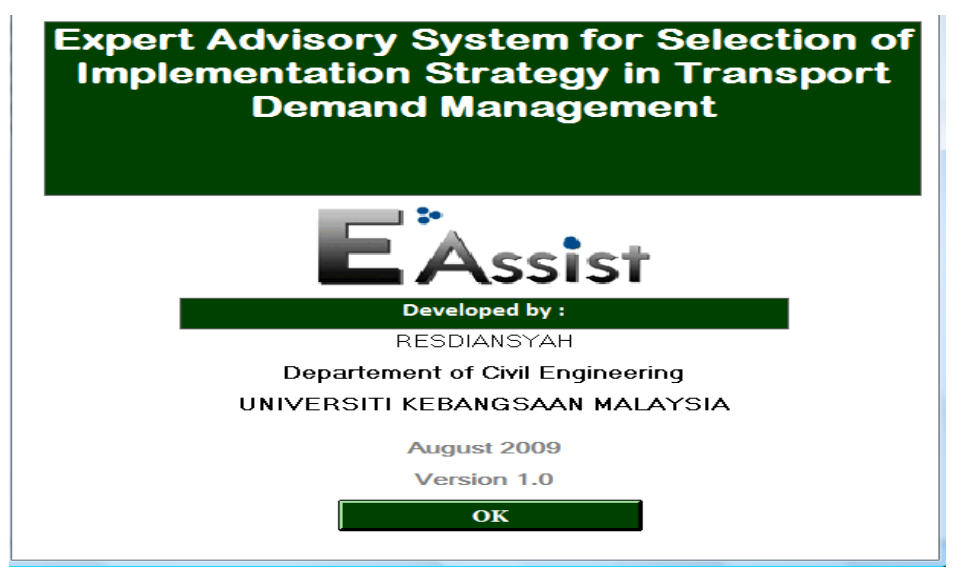

Figure 4 GUI of the rule-based expert system 
By clicking on the OK button closes the general information interface and invokes the main system window that leads the user to nine main objective of implementing TDM, as shown in figure.5. There are nine main objectives associated in this interface as inputs define. Select the one that matches the problem the user encountered in current situation in order to see the full description about the objective, in this case Energy Conservation and Emission Reduction.

Example of this situation is when the new transport planner facing with the "pollution problem" in one area triggered by heavy vehicle such as trucks. To solve this issue, the user needs to select Energy Conservation and Emission Reduction as the input in line with the objective of this strategy to reducing vehicle energy consumption and pollution emission. After the selection, the description of this TDM strategy was appeared on the specific box allocated. If the user confirmed with the input (correct main objective) then clicking on the NEXT button calls up the viewing window that displays the Specific Aim to Achieve the Main Objective interface, as shows in figure 6.

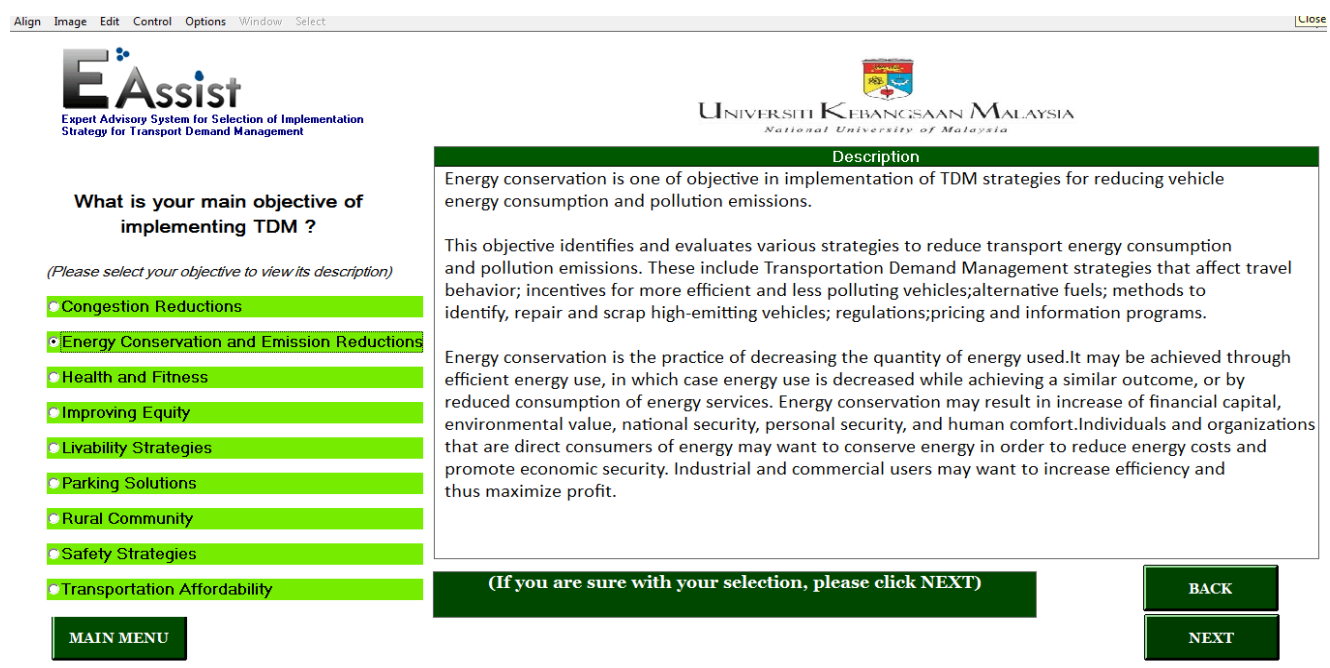

Figure 5 TDM main objective interfaces

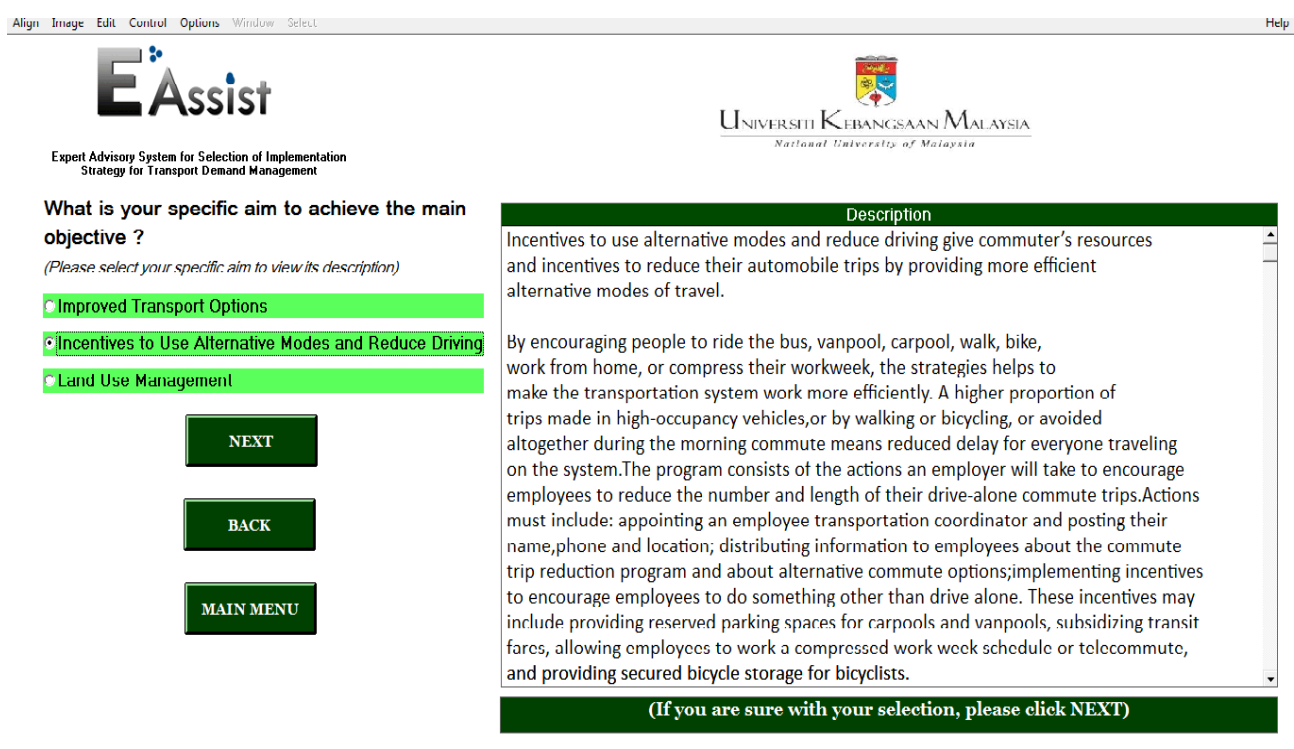

Fig.6 TDM specific aim interface

If the user cannot confirm the description is his/her objective to implement TDM then it is necessary to click BACK button, click on the other main objective as the input, read the TDM main objective description, and do the same step as before, until the actual main objective is found and confirmed. Once interface of Specific Aim appeared, clicking on the bullet button to see the specific aim description to implement the TDM on how to achieve the main objective as shown in previous step.

This dialog window was designed to ensure the user choose appropriate input to avoid wrong advise in appropriate strategy. In this case, user has chosen "Incentives to use Alternative Modes and Reducing Driving" as the Specific Aim to achieve main objective which was described on the dialog box. If the user confirmed with the selection, then clicking on the NEXT button calls up the viewing window that displays the GEOGRAPHICAL AREA interface as shown in figure 7. 


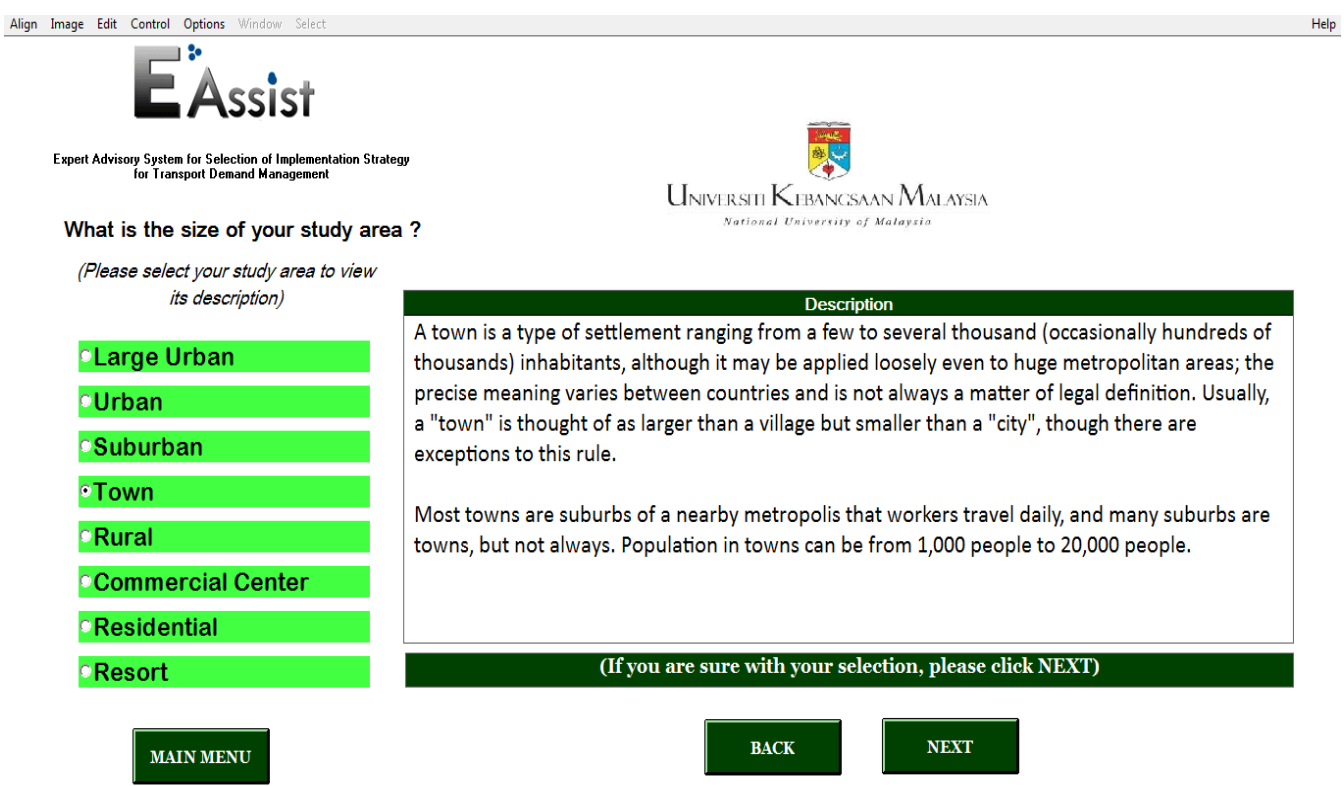

Figure 7 Geographical area interfaces

After selection of specific objective, the sizes of study areas were listed on the left of interface window. The geographical area was identified as important step since all TDM policies in particularly only can be effective in certain area in term of size and population. If the user cannot expect the size of their study area or not sure about the geographical condition in particular area, then they can refer to the description box provided. If the size is identified and selected; for example TOWN, then by click NEXT button will calls up the viewing window that displays the ADVISE ON TDM STRATEGIES as shown in figure 8 below.

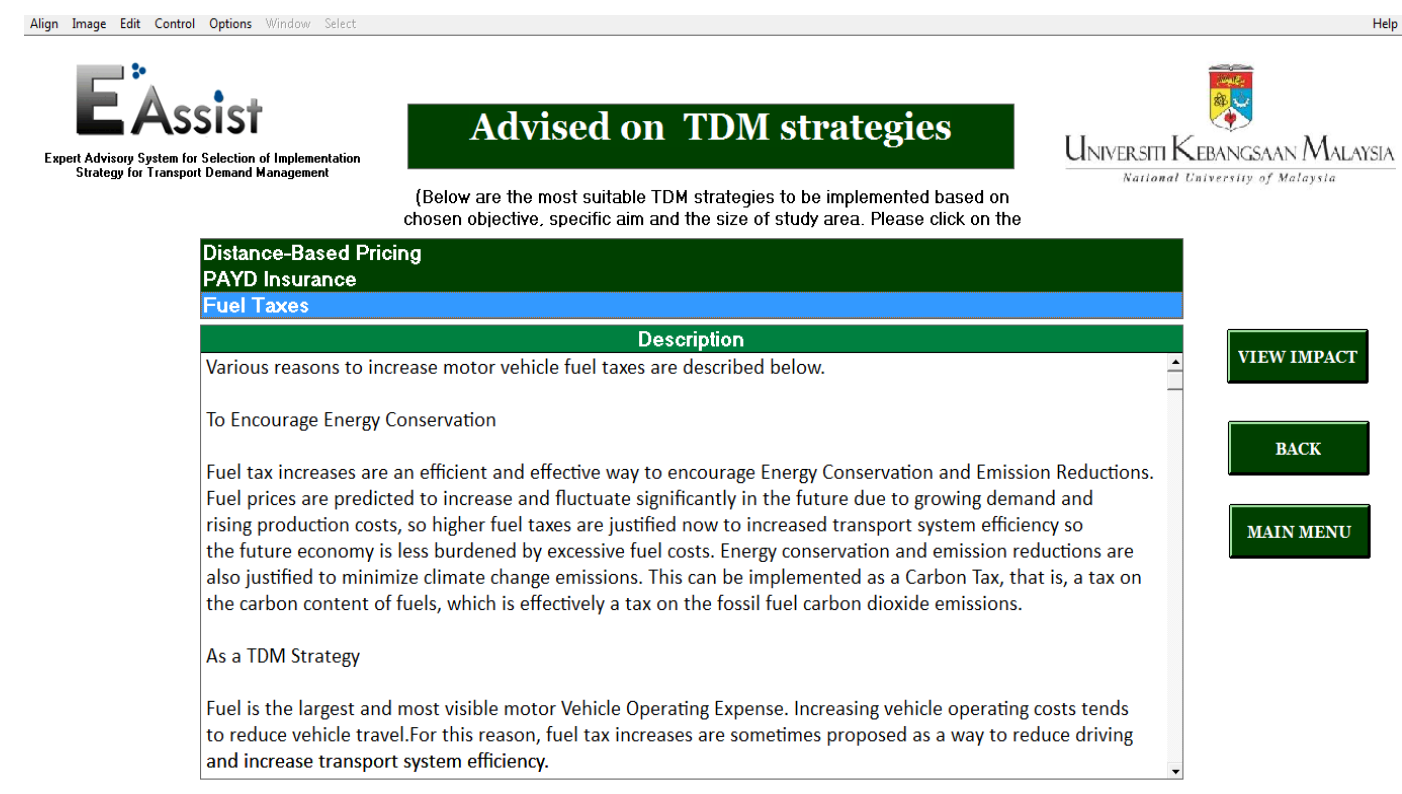

Figure 8 Advised TDM strategies result interface

Fig.8 shows the advice strategy window. It was display three appropriate policies which is the most suitable TDM policies to be implemented based on the input selection. The ES considers three policies for user to judge and make decision of suitable TDM policy. Descriptions for all three policies were provided in box dialog in order to help and guide the user. The user also can click the VIEW IMPACT button and ADVISE STRATEGY IMPACT SUMMARY will display on the interface as shown on figure 9 . 
UNIMAS E-Journal of Civil Engineering, Vol. 4: Issue 1 /April 2013

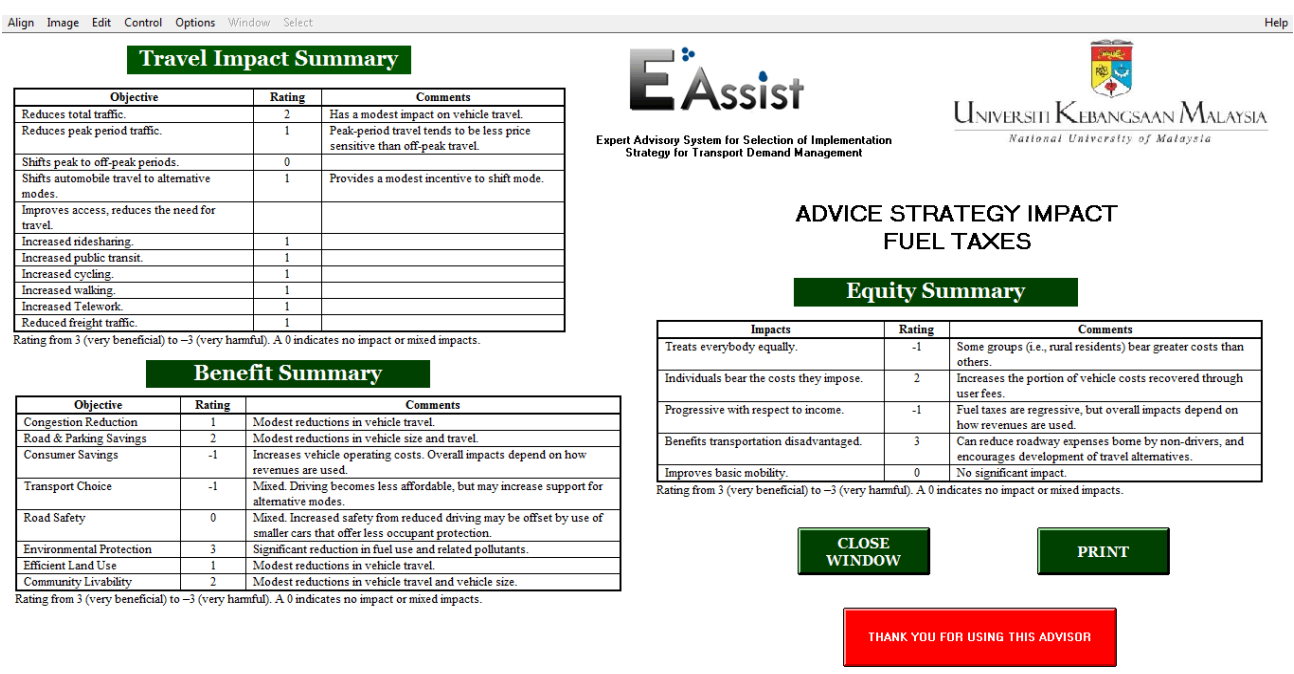

Figure 9 Impact summary interfaces

Figure 9 above shows the advice policies for "fuel tax" as an example. Result shows that travel impact in term of reducing total traffic significantly as rating " 2 " was shown (beneficial). On benefit summary, environmental protecting given rating" 3 " which is significant reduce in fuel use and related pollutants. Advice policies summary shows the travel impact, benefit and equity if the policy chosen will be implemented. Based on the travel impact summary, user can easily to identify how effective reduction of trip or total traffic which is stated in the summary using the rating scale; rating from 3 (very beneficial) to -3 (very harmful); 0 indicates no impact or mixed impact on the objective if user implement this policy. In this case study, the user has identified his objective related to "Energy Efficiency" with specific aim is "Incentives to use Alternative Modes and Reducing Driving"; implementing in TOWN. As result mentioned, three advises were given according to the input selected such as DISTANCE BASED-PRICING, PAYD INSURANCE and FUEL TAX

\section{CONCLUSION AND FUTURE WORK}

The expert system developed is user friendly, and it does not require the user to have any programming knowledge to use it. The user can smoothly navigate through nine different input screens before the system outputs its conclusion. Without referring to any TDM expert, the system can help young and inexperienced engineers and planners in their decision making. In addition, such systems are less costly to use and save time. Moreover, the successful development of an expert system for implementations of TDM demonstrates that the application of rule-based expert system in this domain is promising. Framebased knowledge representation method in conjunction with the power of object-oriented modelling enables the knowledge to be abstracted and represented for the computer to manipulate efficiently and for the knowledge engineer to update the knowledge base with great flexibility.

\section{ACKNOWLEDGMENT}

I would like to thank to Linton University College in Collaboration with University of East London for their support in this research.

\section{REFERENCES}

[1] T. Litman, Are vehicle travel reduction targets justified? Evaluating mobility management policy objectives such as targets to reduce VMT and increase use of alternative modes. Victoria Transport Policy Institute, 2012.

[2] G. Frame. Traffic management and transport demand management. Distance Learning Course in Urban Planning, 2010, Chapter 4, 1-19.

[3] Md. Nazrul Islam, "Development of an expert system for making high performance concrete using hybrid knowledge representation system, “ Ph.D Thesis, Department of Civil Engineering, Universiti Kebangsaan Malaysia, 2004.

[4] Kappa PC version 2.4 user manual, Intellicorp, Inc CA, 2007.

[5] P. Polach, J. Valenta and V. Jirsik, "Hybrid expert system shell," Proceeding of the 4th European Computing Conference. 2010, pp. 148-153, ISBN: 978-960-474-178-6.

[6] R.M. Sonar,"An enterprise intelligent system development and solution framework". World Academy of Science, Engineering and Technology, vol.25, pp.34-39, 2007.

[7] H. Penny Nii, "Tools and infrastructure for knowledge: Based system”, Knowledge Based in Japan. Pp.235-256, Loyola College in Maryland: WTEC Hyper-Librarian, 1993.

[8] K. Darlington, The essence of expert systems, Prentice Hall, 2000.

[9] J.J. Pomykalski and D.E. Brown, "Knowledge-based system design enhancement through reliability measurement, "Expert System with Applications: An International Journal, vol.11, no.3, pp. 277-286, 1996.

[10] J.L. Kaetzel and J.R. Clifton, "Expert knowledge based system for material in construction industry: State of the art report", Journal of Materials and Structures, vol. 28, no. 3, pp.160-174, 1995.

[11] J. Giarrantano and G.D. Riley, Expert system: Principles and Programming, Canada: Thompson Learning, 2005. 